\title{
Updates in Clinical and Genetics Aspects of Hypermobile Ehlers Danlos Syndrome
}

\author{
Irman Forghani
}

Clinic of Clinical and Translational Genetics, Dr. John T. Macdonald Foundation Department of Human Genetics, University of Miami, Miller School of Medicine, Miami, USA

Efforts on recognition, diagnosis, and management of the presumed, most common connective tissue disorder hypermobile Ehlers-Danlos syndrome have been an ongoing challenge, even decades after the description of this condition. A recent international consortium proposed a revised Ehlers-Danlos syndrome classification, an update much needed since Villefranche nosology, in 1998. Hypermobile Ehlers-Danlos syndrome is the only subtype in these groups of syndromes with no known genetic cause(s). This effort brought significant attention to this often underappreciated condition. This review provides an update of the clinical and genetic aspects of hypermobile Ehlers-Danlos syndrome for clinicians and researchers. Keywords: Ehlers-Danlos syndrome, classification, joint hypermobility, hypermobility spectrum disorder
Ehlers-Danlos syndromes (EDS) are a clinically and genetically heterogeneous group of heritable connective tissue disorders characterized by joint hypermobility $(\mathrm{JH})$, skin hyperextensibility, and tissue fragility (1). EDS were first described by Hippocrates in $400 \mathrm{BC}$ as a condition with joint laxity and multiple scars $(2,3)$. In 1892, the Russian dermatologist Dr. Tschernogubow published the first comprehensive description of the syndrome (2,3). In 1901, the Danish dermatologist Edvard Ehlers and in 1908, the French dermatologist Henri-Alexandre Danlos each further defined the syndrome as a distinct entity. Eventually, in 1936, the English dermatologist Frederick Parkes-Weber suggested the name EDS $(2,3)$. The first description of JH syndrome (JHS) was published in 1967 by Kirk et al. (4). Further classification of EDS into subgroups and delineation of hypermobile EDS (hEDS) are discussed below. The 2017 classification describes 13 subtypes of EDS, with hEDS being the most common subtype $(1,5)$. hEDS is predominantly characterized by generalized $\mathrm{JH}(\mathrm{GJH})$, occurring within the first years of life, related musculoskeletal complications, mild skin hyperextensibility, mild aortic root dilatation, mitral valve prolapse, and easy bruising (5-7). Skin hyperextensibility in hEDS is milder than that in other forms of EDS such as classical EDS (cEDS) or classical-like EDS (clEDS). Other features associated with this syndrome include chronic pain, functional gastrointestinal disorders, Postural Orthostatic Tachycardia syndrome, and psychological dysfunction $(1,5,6)$. These associated syndromes can be as debilitating as secondary complications of joint laxity $(5,6)$. The diagnosis of hEDS is clinical and is established through a physical examination and a review of medical and family histories $(1,5,6)$. The patients usually present with chronic pain, chronic fatigue, recurrent joint subluxations and dislocations, dysautonomia, functional gastrointestinal problems, and anxiety $(5,6)$. A majority of the patients visit several specialists, seeking a diagnosis. hEDS behaves as an autosomal dominant syndrome (7). Some authors have reported a complete penetrance, whereas others observed incomplete penetrance and variable expressivity $(5,6)$. One study on a large family proposed a $90 \%$ penetrance (8). The incidence of hEDS is higher among females than among males, and it is more commonly found in some populations such as Africans than in Caucasians $(9,10)$. Castori and his group suggested that female predominance is related to differences in muscle pain perception related to the effects of sex hormones (9). Relevant to this topic, the effects of estrogen and estrogen-like compounds in the homeostasis of connective tissue and the knowledge gap in this area have been recently proposed, thereby opening an interesting area of research $(11,12)$.

\section{Ehlers-Danlos Syndrome Classification}

The first classification of EDS was established in the 1960s (3). In 1988, Beighton et al. (13) published a revised classification known as the "Berlin nosology," which was proposed at the seventh International Congress of Human Genetics in Berlin in 1986. In the Berlin nosology, 11 EDS subtypes were defined, primarily based on the mode of inheritance and clinical presentations. They

Address for Correspondence: Irman Forghani, Division of Clinical and Translational Genetics, Dr. John T. Macdonald Foundation Department of Human Genetics, University of Miami, Miller School of Medicine, Miami, USA

e-mail: IForghani@med.miami.eduＯRCID ID: orcid.org/0000-0002-5484-5186

Received: 26 June $2018 \quad$ Accepted: 27 July 2018 • DOI: 10.4274/balkanmedj.2018.1113

Available at www.balkanmedicaljournal.org

Cite this article as:

Forghani I. Updates in Clinical and Genetics Aspects of Hypermobile Ehlers Danlos Syndrome. Balkan Med J 2019;36:12-6

${ }^{\circ}$ Copyright 2019 by Trakya University Faculty of Medicine / The Balkan Medical Journal published by Galenos Publishing House. 
designated a Roman numeral to each subtype (14). In 1997, with further elucidation of the molecular causes of each EDS subtype, the Villefranche nosology stemmed from a meeting of experts in this field at Villefranche-Sur-Mer in France. The Villefranche nosology was published by Beighton et al. (14) in 1998. This nosology delineated six major subtypes for EDS and described the major and minor criteria for each subtype. Recently, an independent group of experts in this field created the international EDS consortium, and their efforts resulted in the latest classification of EDS in 2017 (1). Their proposed nosology is based on characteristic clinical manifestations and molecular and causative genetic variants in each EDS subtype; the sole exception is the hEDS subtype, the molecular cause of which remains unknown. The consortium defined 13 EDS subtypes, which are caused by pathogenic variants in 19 different genes, and revised the diagnostic criteria for hEDS $(1,15)$. They proposed their nosology using a new nomenclature, emphasizing the description of each subtype, and designated a descriptive name and acronym to each subtype. Therefore, what was previously known as Ehlers-Danlos hypermobility or type III EDS is now identified as hypermobile EDS or hEDS.

\section{Diagnosis of Hypermobile Ehlers-Danlos Syndrome Based on the 2017 Diagnostic Criteria}

Despite the significant advancements in molecular genetics, efforts toward the discovery of the genetic cause(s) of hEDS have yielded inconclusive results. The lack of success in identifying the molecular etiology of this condition is due to the heterogeneity and the variable expressivity of the condition, as well as the nonspecific phenotypes that overlap with other heritable connective tissue disorder and non-heritable disorders of connective tissue (1). The Villefranche nosology did not clearly define the required inclusive and exclusive criteria for establishing the diagnosis of hEDS. The criteria were divided into major and minor, with the major criteria including skin hyperextensibility and GJH and the minor criteria being chronic pain, recurrent joint dislocation, and positive family history. GJH refers to hypermobility of at least five or more joints, at the same time, usually at four limbs and the spine $(16,17)$. The 2017 diagnostic criteria for hEDS are more specific and exclusive. A useful checklist of diagnostic criteria for hEDS is available on the EDS society website (https://ehlers-danlos.com/wp-content/ uploads/hEDS-Dx-Criteria-checklist-1.pdf). The DazzleVegas 2017 Global Learning Conference videos presented by the EDS society provide detailed discussions about the new diagnostic criteria and their proper implementation (https://www.ehlers-danlos.com/2017eds-global-conference/). Fulfillment of the following three criteria concurrently is required for establishing the hEDS diagnosis (1):

- Criterion 1 is the presence of GJH.

- Criterion 2 consists of three separate features (A, B, and C); feature $\mathrm{A}$ is systemic manifestations related to heritable connective tissue disorder, feature B is a positive family history, and feature $\mathrm{C}$ includes pain and secondary musculoskeletal complications of joint laxity.

- Criterion 3 is for the exclusion of other heritable and acquired causes of hypermobility and possible alternative diagnoses that can present with $\mathrm{JH}$.
Criterion 1: The Beighton scoring system, which was established in 1973, continues to be the most reliable assessment tool for GJH $(16,17)$. A Beighton score ranges from 0 to 9 (Table 1) and is influenced by age, sex, ethnicity, history of trauma, and physical fitness of the person $(16,17)$. Furthermore, for conditions that affect the Beighton score, such as previous surgeries and amputation of the joint, a self-reported five-point questionnaire [(5PQ), Table 2] is used to consider historic hypermobility $(18,19)$. The consortium defined an age-adjusted cut-off value to consider the age-related changes in GJH. A cut-off point of $\geq 4$ for men and women aged $>50$ years, a cut-off point of $\geq 5$ for adults aged till 50 years, and a cutoff point of $\geq 6$ for prepubertal children and adolescents confirm the presence of GJH. In addition, to take the historic JH into account, the committee decided to add one point to the Beighton score, when it is one point below the cut-off point and if two or more items in the 5PQ are positive (1). Experts recommend using an orthopedic goniometer to increase the accuracy of the GJH assessment (https:// www.ehlers-danlos.com/assessing-joint-hypermobility/). Criterion 2 (features A-C). At least two of the features must be positive in the patient to meet criterion 2 (1).

- Feature A (Table 3) requires at least five positive items.

- Feature B is a positive family history (one or more first-degree family members who meet the 2017 diagnostic criteria for hEDS; therefore, the reported family history requires further investigation). - Feature $\mathrm{C}$ at least one must be positive, including daily musculoskeletal pain in $\operatorname{limb}(\mathrm{s})$, for 3 or more months, and/or chronic generalized pain for 3 or more months, and/or recurrent joint dislocation or frank joint instability not caused by trauma. It should be noted that feature $\mathrm{C}$ cannot be counted for those patients who have been previously diagnosed with an autoimmune disorder; instead, they must fulfill features A and B. Criterion 3 is aimed at the exclusion of other connective tissue disorders. All the following three prerequisites must be met: the absence of unusual skin fragility

TABLE 1. Beighton scoring system (14) 1. Passive dorsiflexion of fifth finger beyond 90 One point for each side
degrees

2. Bilateral apposition of the thumb to the flexor aspect of the forearm

3. Hyperextension of elbows, beyond 10 degrees One point for each side

4. Hyperextension of knees, beyond 10 degrees One point for each side

5. The palm of hands can be placed fully flat on the floor with forward bending of the trunk with One point locked straight knees and feet together

TABLE 2. 5-point questionnaire (18)

1. Can you now (or could you ever) place your hands flat on the floor without bending your knees?

2. Can you now (or could you ever) bend your thumb to touch your forearm?

3. As a child did you amuse your friends by contorting your body into strange shapes odds ratio could you do the splits?

4. As a child or teenager did your shoulder or kneecap dislocate on more than one occasion?

5. Do you consider yourself double-jointed? 
(consider other forms of EDS in the presence of unusual skin fragility), the exclusion of other heritable and acquired (autoimmune disorders) connective tissue disorders, and the exclusion of other heritable causes of $\mathrm{JH}$. Molecular testing, which is not recommended for the diagnosis of hEDS, can be considered for the exclusion of other heritable causes of JH if there are any clinical concerns (1).

\section{Hypermobility Spectrum Disorder}

In 1999, Grahame proposed that benign or asymptomatic JH and JHS are possibly the two ends of one spectrum (20). Furthermore, several experts have suggested that JHS and hEDS, which were initially introduced as two separate clinical entities, were actually equivalent conditions $(21,22)$. Recently, Castori of experts elegantly described a dynamic framework for the classification of $\mathrm{JH}$ and the genetic syndromes featuring $\mathrm{JH}$ without a known molecular etiology. They merged these conditions into one umbrella of diagnosis and introduced the new terminology of hypermobility spectrum disorders that fills the gap between asymptomatic $\mathrm{JH}$ and hEDS. Hypermobility spectrum disorder includes all the conditions featuring $\mathrm{JH}$ plus one or more of its secondary musculoskeletal manifestations and is a diagnosis of exclusion for patients with symptomatic JH who do not meet the diagnostic criteria for other types of EDS or hEDS (23). It was indisputable that embodiment of a more selective diagnostic criteria for hEDS would exclude many of the individuals with symptomatic JH. Hypermobility spectrum disorder is an alternative diagnosis for this group of patients.

\section{Genetic Research on Hypermobile Ehlers-Danlos Syndrome}

Creating a more homogeneous cohort for scientific research was one of the primary goals of the consortium in refining

TABLE 3. Feature A, Criterion 2, the 2017 diagnostic criteria for hypermobile Ehlers-Danlos syndromes (1)

\footnotetext{
1. Unusually soft or velvety skin

2. Mild skin hyperextensibility

3. Unexplained striae such as striae distensae or rubrae at the back, groins, thighs, breasts and/or abdomen in adolescents, men or prepubertal women without a history of significant gain or loss of body fat or weight

4. Bilateral piezogenic papules of the heel

5. Recurrent or multiple abdominal hernia(s) (e.g., umbilical, inguinal, crural)
}

6. Atrophic scarring involving at least two sites and without the formation of truly papyraceous and/or hemosideric scars as seen in classical EhlersDanlos syndromes

7. Pelvic floor, rectal, and/or uterine prolapse in children, men or nulliparous women without a history of morbid obesity or other known predisposing medical condition

8. Dental crowding and high or narrow palate

9. Arachnodactyly, as defined in one or more of the followings: (i) positive wrist sign (Steinberg sign) on both sides; (ii) positive thumb sign (Walker sign) on both sides

10. Arm span-to-height $\geq 1.05$

11. Mitral valve prolapse mild or greater based on strict echocardiographic criteria

12. Aortic root dilatation with Z-score $>+2$ the diagnostic criteria for hEDS $(1,23)$. Previous and recent studies favor the clinical and genetic heterogeneity of this syndrome, making the identification of its genetic etiology more difficult $(24,25)$. A chronological review of the relevant studies is presented below. In 1994, Narcisi et al. (26) reported the first mutation in five affected members of a family with clinical characteristics of hEDS who carried a pathogenic variant in the COL3A1 gene. None of them had history of vascular fragility, characteristic facial features, or any other characteristics of vascular EDS (vEDS). This COL3A1 variant has not been reported in any other cases of hEDS and is noted in the ClinVar database as EDS, Nonvascular variant [NM_000090.3(COL3A1):c.2410G>A (p.Gly804Ser)] (https:// www.ncbi.nlm.nih.gov/clinvar/variation/17221/). A 2004 twin study on 483 monozygotic and 472 dizygotic female twin pairs, conducted in the UK, reported an estimated heritability of $70 \%$ and a higher concordance for $\mathrm{JH}$ in the monozygotic twins (60\%) than in the dizygotic twins (36\%), suggesting that JH has a highly significant genetic component (27). In 2001, Schalkwijk et al. (28) introduced a subtype of EDS that is now known as clEDS. clEDS is an autosomal recessive form of EDS and is caused by a deficiency of tenascin-XB, an extracellular matrix glycoprotein encoded by the $T N X B$ gene. The same group later described an association between haploinsufficiency of the TNXB gene and $\mathrm{JH}$ in heterozygous females. They evaluated 20 heterozygous family members comprising 14 females and 6 males. All the subjects showed a significant decrease in serum tenascin levels, although 9 of them, all females, showed GJH, when evaluated using the Beighton scoring system. Despite this reduction in serum tenascin levels, none of the heterozygous males presented with JH (29). In 2013, Merke et al. (30) described a contiguous gene deletion syndrome caused by deletion of CYP21A2 and its flanking gene $T N X B$, and they termed it as congenital adrenal hyperplasia-X syndrome. Congenital adrenal hyperplasia-X syndrome presents with combined phenotypes of congenital adrenal hyperplasia and hypermobility. The authors associated the hypermobility phenotype of this syndrome to haploinsufficiency of the TNXB gene (30). In 2015, Morissette et al. (31) found a heterozygous missense variant, c. $12174 \mathrm{C}>\mathrm{G}$ (p.C4058W), in the $T N X B$ gene in 10 people from seven families with congenital adrenal hyperplasia-X. They showed that this variant does not affect the protein expression of tenascin in dermal fibroblasts and proposed a dominant-negative mechanism for this missense variant, which is different from the haploinsufficiency caused by deletion or truncated mutations previously reported. The molecular analysis of the $T N X B$ gene is challenging due to the presence of a pseudogene. Most of the available gene panels do not include this gene, which may impact the real estimate of the frequency of this gene in patients with hEDS. In 2015, Syx et al. (8) performed the first genome-wide linkage analysis in a large Belgium family with hEDS and suggested an $8.8-\mathrm{Mb}$ candidate linkage interval on chromosome 8 (8p22-8p21.1), with a maximum two-point LOD score of 4.73. Whole exome sequencing of two affected family members subsequently identified a missense variant in the leucine zipper, putative tumor suppressor-1 gene (LZTS1) in this region (p.His211Gln). 
All the affected, and none of the unaffected, individuals in this family harbored the same variant in LZTS1. However, the authors did not find the 8p22-8p21.1 locus linkage in four other hEDS families. Interestingly, sequencing of the LZTS1 gene in 230 additional unrelated individuals with hEDS identified three additional variants in this gene (8). They concluded that LZTS1 variants are associated with a small percentage of patients with hEDS ( $2 \%$ in their cohort), thereby confirming the genetic heterogeneity of this disorder.

In 2016, at the University of Brescia, Italy, Colombi and her group conducted an in vitro comprehensive immunofluorescence analysis and transcriptome-wide expression profiling using cultured fibroblasts of five affected females with JHS and hEDS (32). Their study demonstrated disorganization of collagens and fibronectin and their integrin receptors, along with a widespread disarray of several matrix structural components (32). Furthermore, they demonstrated altered expression of multiple genes involved in homeostasis signaling cascades, maintenance of extracellular matrix architecture, cell-cell adhesion, and inflammatory/ immune/pain responses. This observation in hEDS/hypermobility spectrum disorder was consistent with their previous findings in cEDS, vEDS, and the majority of other EDS types (33). In addition, they reported overexpression of an alternative fibronectin (av $\beta 3$ integrin) receptor in the fibroblasts of patients with hEDS or JHS similar to that in patients with cEDS and vEDS $(32,33)$. They had previously proposed the compensatory role of the av $\beta 3$ integrin receptor in anoikis (apoptosis induced by extracellular matrix disassembly) rescue in COL5A1 and COL $3 A 1$ mutations associated with cEDS and vEDS, respectively (33). These studies agree with previous works describing the clinical and genetic heterogeneity of this syndrome (8). The same group recently published a follow-up study regarding cellular characterization of dermal fibroblasts in patients with hEDS or JHS (34). They described an exclusive myofibroblast-like phenotype in in vitro dermal fibroblasts of patients with hEDS/hypermobility spectrum disorder, in contrast to other types of EDS, and the role of the av $\beta 3$ integrin receptor in this phenotype, in addition to the anoikis rescue. They also hypothesized that a constellation of factors such as persistent expression of the alpha-smooth muscle actin cytoskeleton, as well as high levels of the active form of matrix metallopeptidase-9, along with the role of inflammatory mediators and altered expression of $\mathrm{N}$-cadherin family could be involved in the pathogenesis of hEDS/hypermobility spectrum disorder and the formation of the myofibroblast-like phenotype $(32,34)$. They suggested that matrix metallopeptidase-9, which is involved in the physiological degradation of the extracellular matrix, plays a role in fibronectin fragmentation and extracellular matrix disassembly in patients with hEDS/hypermobility spectrum disorder (34). Alpha-smooth muscle actin cytoskeleton is expressed during wound healing and tissue regeneration and is required for the physiological transition of fibroblasts to myofibroblasts (35). Furthermore, Colombi and her group proposed a regulatory effect of cadherin on the expression of alpha-smooth muscle actin cytoskeleton through the $\mathrm{Wnt} / \mathrm{b}$-catenin pathway, the involvement of av $\beta 3$ integrin-ILK-mediated signal transduction and Snail/Slug in the overexpression of matrix metallopeptidase-9, disorganization of alpha-smooth muscle actin cytoskeleton and fibroblast to myofibroblast transition, and subsequently, the pathogenesis of hEDS or hypermobility spectrum disorder. Interestingly, they observed the myofibroblast-like phenotype in control cells grown in conditioned media of hEDS/hypermobility spectrum disorder cultures, suggesting the proteolytic activity of conditioned media in the myofibroblast-like phenotype, similar to a chronic inflammatory state. Therefore, they suggested that this multisystem disorder is a part of a sequence of altered extracellular matrix and chronic inflammatory state, whereas a a distinct clinical entity (34). In 2018, Colombi and her group summarized their work about the key role of av $\beta 3$ integrin and its different role through specific signaling pathways in each EDS subtype based on their molecular basis (36). However, a better understanding of these complex pathways is needed to elucidate the pathogenesis of these conditions.

\section{Future Directions}

The exact prevalence of hEDS is not clear (37). Application of the recent diagnostic criteria and clear exclusion of hEDS from hypermobility spectrum disorder entail future observational studies for achievement of an accurate prevalence of these conditions. Determination of GJH in the new diagnostic criteria for hEDS is adjusted according to the age. However, the implication of age into other recently introduced criteria is not clear. Some phenotypic features of hereditary connective tissue disorders emerge as age progresses. Longitudinal studies are needed for better understanding of the temporal nature of hEDS and hypermobility spectrum disorder. Recent studies on cultured fibroblasts from a small number of adult females with hEDS were suggestive of an inflammatory-like condition and "a phenotypic continuum rather than a distinct clinical entity" (32). Further studies on younger individuals diagnosed with hEDS or hypermobility spectrum disorder would help in distinguishing between secondary vs primary inflammatory state. The prevalence of JHS is estimated to be around $2 \%$, and a twin study suggested a heritability of $70 \%$ $(27,37)$. Similarly, the prevalence and heritability of schizophrenia are $1 \%$ and $80 \%$, respectively $(38,39)$. Genome-wide association studies in schizophrenia were successful in identifying multiple gene loci and candidate genes $(40,41)$. A multicentric genome-wide association study on hypermobility spectrum disorder and hEDS could identify novel candidate loci in this spectrum of disorders.

Conflict of Interest: No conflict of interest was declared by the authors.

Financial Disclosure: No financial disclosure was declared by the authors.

\section{REFERENCES}

1. Malfait F, Francomano C, Byers P, Belmont J, Berglund B, Black J. et al. The 2017 international classification of the Ehlers-Danlos syndromes. Am J Med Genet C Semin Med Genet 2017;175:8-26.

2. Enersen OD. Ehlers-Danlos syndrome. Oslo, Norway: who named it? (2004). Available from: http://www.whonamedit.com/synd.cfm/2017.html

3. Parapia LA, Jackson C. Ehlers-Danlos syndrome a historical review. Br J Haematol 2008;141:32-5.

4. Kirk JA, Ansell BM, Bywaters EG. The hypermobility syndrome. Musculoskeletal complaints associated with generalized joint hypermobility. Ann Rheum Dis $1967 ; 26: 419-25$. 
5. Castori M. Ehlers-danlos syndrome, hypermobility type: an underdiagnosed hereditary connective tissue disorder with mucocutaneous, articular, and systemic manifestations. ISRN Dermatol 2012;2012:751768.

6. Tinkle B, Castori M, Berglund B, Cohen H, Grahame R, Kazkaz H, et al. Hypermobile Ehlers-Danlos syndrome (a.k.a. Ehlers-Danlos syndrome Type III and Ehlers-Danlos syndrome hypermobility type): Clinical description and natural history. Am J Med Genet C Semin Med Genet 2017;175:48-69.

7. Levy HP. Hypermobile Ehlers-Danlos Syndrome. GeneReviews 1993.

8. Syx D, Symoens S, Steyaert W, De Paepe A, Coucke PJ, Malfait F. Ehlers-Danlos Syndrome, Hypermobility Type, Is Linked to Chromosome 8p22-8p21.1 in an Extended Belgian Family. Dis Markers 2015;2015:828970.

9. Castori M, Camerota F, Celletti C, Grammatico P, Padua L. Ehlers-Danlos syndrome hypermobility type and the excess of affected females: possible mechanisms and perspectives. Am J Med Genet A 2010;152:2406-8.

10. Remvig L, Jensen DV, Ward RC. Epidemiology of general joint hypermobility and basis for the proposed criteria for benign joint hypermobility syndrome: review of the literature. J Rheumatol 2007;34:804-9.

11. Boyan BD, Hart DA, Enoka RM, Nicolella DP, Resnick E, Berkley KJ, et al. Hormonal modulation of connective tissue homeostasis and sex differences in risk for osteoarthritis of the knee. Biol Sex Differ 2013;4:3.

12. Leblanc DR, Schneider M, Angele P, Vollmer G, Docheva D. The effect of estrogen on tendon and ligament metabolism and function. J Steroid Biochem Mol Biol 2017;172:106-16.

13. Beighton P, de Paepe A, Danks D, Finidori G, Gedde-Dahl T, Goodman R, et al. International Nosology of Heritable Disorders of Connective Tissue, Berlin, 1986. Am J Med Genet 1988;29:581-94.

14. Beighton P, De Paepe A, Steinmann B, Tsipouras P, Wenstrup RJ. Ehlers-Danlos syndromes: revised nosology, Villefranche, 1997. Ehlers-Danlos National Foundation (USA) and Ehlers-Danlos Support Group (UK). Am J Med Genet 1998;77:31-7.

15. Castori M, Hakim A. Contemporary approach to joint hypermobility and related disorders. Curr Opin Pediatr 2017;29:640-9.

16. Beighton P, Solomon L, Soskolne CL. Articular mobility in an African population. Ann Rheum Dis 1973;32:413-8.

17. Scheper MC, Engelbert RH, Rameckers EA, Verbunt J, Remvig L, Juul-Kristensen B. Children with generalised joint hypermobility and musculoskeletal complaints: state of the art on diagnostics, clinical characteristics, and treatment. Biomed Res Int 2013;2013:121054.

18. Hakim A, Grahame R. Joint hypermobility. Best Pract Res Clin Rheumatol 2003; 17:989-1004.

19. Juul-Kristensen B, Schmedling K, Rombaut L, Lund H, Engelbert RH. Measurement properties of clinical assessment methods for classifying generalized joint hypermobility-A systematic review. Am J Med Genet C Semin Med Genet 2017;175:116-47.

20. Grahame R. Joint hypermobility and genetic collagen disorders: are they related? Arch Dis Child 1999;80:188-91.

21. Ross J, Grahame R. Joint hypermobility syndrome. BMJ 2011;342:7167.

22. Castori M, Dordoni C, Valiante M, Sperduti I, Ritelli M, Morlino S, et al. Nosology and inheritance pattern(s) of joint hypermobility syndrome and Ehlers-Danlos syndrome, hypermobility type: a study of intrafamilial and interfamilial variability in 23 Italian pedigrees. Am J Med Genet A 2014;164:3010-20.

23. Castori M, Tinkle B, Levy H, Grahame R, Malfait F, Hakim A. A framework for the classification of joint hypermobility and related conditions. Am J Med Genet C Semin Med Genet 2017;175:148-57.
24. De Wandele I, Rombaut L, Malfait F, De Backer T, De Paepe A, Calders P. Clinical heterogeneity in patients with the hypermobility type of Ehlers-Danlos syndrome. Res Dev Disabil 2013;34:873-81.

25. Tinkle BT, Bird HA, Grahame R, Lavallee M, Levy HP, Sillence D. The lack of clinical distinction between the hypermobility type of Ehlers-Danlos syndrome and the joint hypermobility syndrome (a.k.a. hypermobility syndrome). Am J Med Genet A 2009;149:2368-70.

26. Narcisi P, Richards AJ, Ferguson SD, Pope FM. A family with Ehlers-Danlos syndrome type III/articular hypermobility syndrome has a glycine 637 to serine substitution in type III collagen. Hum Mol Genet 1994;3:1617-20.

27. Hakim AJ, Cherkas LF, Grahame R, Spector TD, MacGregor AJ. The genetic epidemiology of joint hypermobility: a population study of female twins. Arthritis Rheum 2004;50:2640-4.

28. Schalkwijk J, Zweers MC, Steijlen PM, Dean WB, Taylor G, van Vlijmen IM, et al A recessive form of the Ehlers-Danlos syndrome caused by tenascin-X deficiency. $\mathrm{N}$ Engl J Med 2001;345:1167-75.

29. Zweers MC, Bristow J, Steijlen PM, Dean WB, Hamel BC, Otero M, et al. Haploinsufficiency of TNXB is associated with hypermobility type of Ehlers-Danlos syndrome. Am J Hum Genet 2003;73:214-7.

30. Merke DP, Chen W, Morissette R, Xu Z, Van Ryzin C, Sachdev V, et al. Tenascin-X haploinsufficiency associated with Ehlers-Danlos syndrome in patients with congenital adrenal hyperplasia. J Clin Endocrinol Metab 2013;98:379-87.

31. Morissette R, Chen W, Perritt AF, Dreiling JL, Arai AE, Sachdev V, et al. Broadening the Spectrum of Ehlers Danlos Syndrome in Patients With Congenital Adrenal Hyperplasia. J Clin Endocrinol Metab 2015;100:1143-52.

32. Chiarelli N, Carini G, Zoppi N, Dordoni C, Ritelli M, Venturini M, et al Transcriptome-Wide Expression Profiling in Skin Fibroblasts of Patients with Joint Hypermobility Syndrome/Ehlers-Danlos Syndrome Hypermobility Type. PLoS One 2016;11:0161347.

33. Zoppi N, Gardella R, De Paepe A, Barlati S, Colombi M. Human fibroblasts with mutations in COL5A1 and COL3A1 genes do not organize collagens and fibronectin in the extracellular matrix, down-regulate alpha2beta1 integrin, and recruit alphavbeta3 Instead of alpha5beta1 integrin. J Biol Chem 2004;279:18157-68.

34. Zoppi N, Chiarelli N, Binetti S, Ritelli M, Colombi M. Dermal fibroblast-tomyofibroblast transition sustained by $\alpha v \beta 3$ integrin-ILK-Snail1/Slug signaling is a common feature for hypermobile Ehlers-Danlos syndrome and hypermobility spectrum disorders. Biochim Biophys Acta Mol Basis 2018;1864:1010-23.

35. Darby IA, Laverdet B, Bonté F, Desmoulière A. Fibroblasts and myofibroblasts in wound healing. Clin Cosmet Investig Dermatol 2014;7:301-11.

36. Zoppi N, Chiarelli N, Ritelli M, Colombi M. Multifaced Roles of the $\alpha v \beta 3$ Integrin in Ehlers-Danlos and Arterial Tortuosity Syndromes' Dermal Fibroblasts. Int J Mol Sci 2018:19.

37. Hakim AJ, Sahota A. Joint hypermobility and skin elasticity: the hereditary disorders of connective tissue. Clin Dermatol 2006;24:521-33.

38. Schizophrenia Working Group of the Psychiatric Genomics Consortium. Biological insights from 108 schizophrenia-associated genetic loci. Nature 2014;511:421-7.

39. Sullivan PF, Kendler KS, Neale MC. Schizophrenia as a complex trait: evidence from a meta-analysis of twin studies. Arch Gen Psychiatry 2003;60:1187-92.

40. Schizophrenia Psychiatric Genome-Wide Association Study (GWAS) Consortium. Genome-wide association study identifies five new schizophrenia loci. Nat Genet 2011;43:969-76.

41. Lichtenstein P, Björk C, Hultman CM, Scolnick E, Sklar P, Sullivan PF. Recurrence risks for schizophrenia in a Swedish national cohort. Psychol Med 2006;36:1417-25. 